\title{
Quaternions for regularizing Celestial Mechanics The right way
}

\section{Conference Paper}

Author(s):

Waldvogel, Jörg

Publication date:

2008

Permanent link:

https://doi.org/10.3929/ethz-b-000069851

Rights / license:

In Copyright - Non-Commercial Use Permitted

Originally published in:

Celestial mechanics \& dynamical astronomy 102(1-3), https://doi.org/10.1007/s10569-008-9124-y 


\title{
Quaternions for regularizing Celestial Mechanics: the right way
}

\author{
Jörg Waldvogel
}

Received: 7 December 2007 / Revised: 5 February 2008 / Accepted: 8 February 2008 /

Published online: 18 March 2008

(C) Springer Science+Business Media B.V. 2008

\begin{abstract}
Quaternions have been found to be the ideal tool for describing and developing the theory of spatial regularization in Celestial Mechanics. This article corroborates the above statement. Beginning with a summary of quaternion algebra, we will describe the regularization procedure and its consequences in an elegant way. Also, an alternative derivation of the theory of Kepler motion based on regularization will be given. Furthermore, we will consider the regularization of the spatial restricted three-body problem, i.e. the spatial generalization of the Birkhoff transformation. Finally, the perturbed Kepler motion will be described in terms of regularized variables.
\end{abstract}

Keywords Quaternions · Regularization · Kustaanheimo-Stiefel transformation ·

Kepler formulas · Birkhoff transformation · Perturbed Kepler problem · Joukowsky-Birkhoff mapping · Quaternion algebra

\section{Introduction}

In 1844 the Irish mathematician William Rowan Hamilton (1805-1865) published a paper entitled On quaternions, or a new system of imaginaries in algebra (Hamilton 1844). Hamilton got inspiration from two multiplicative operations involving vectors $\in \mathbb{R}^{3}$ (the scalar product and vector product) and managed to devise a non-commutative algebra of four-dimensional objects generalizing the algebra of complex numbers. Quaternions soon became a standard topic in higher analysis, and today, they are in use in computer graphics, control theory, signal processing, orbital mechanics, etc., mainly for representing rotations and orientations in 3-space.

The use of quaternions for the purpose of regularization of the spatial Kepler problem has been contemplated soon after the discovery of the so-called KS transformation

Dedicated to Claude Frœschlé.

J. Waldvogel $(\bowtie)$

Swiss Federal Institute of Technology ETH, 8092 Zurich, Switzerland

e-mail:waldvogel@sam.math.ethz.ch 
by Kustaanheimo and Stiefel (1965). The fact that the KS transformation is based on a four-dimensional parametric space immediately called for bringing quaternions into play. However, in their comprehensive text Stiefel and Scheifele (1971) clearly rejected this idea (p. 286): "Any attempt to substitute the theory of the KS matrix by the more popular theory of the quaternion matrices leads to failure or at least to a very unwieldy formalism." This statement was first refuted by Chelnokov (1981) who presented a regularization theory of the spatial Kepler problem using geometrical considerations in a rotating coordinate system and quaternion matrices. In a series of papers, including Chelnokov $(1992,1999)$, the same author extended the theory of quaternion regularization and also presented practical applications.

Later, but independently, Vivarelli (1983) and Vrbik (1994, 1995) demonstrated the usefulness of quaternions for regularization in celestial mechanics. Recently, the Space Mechanics Group of the University of Zaragoza (Spain) took advantage of the elegance of the quaternion language in various applications in orbital and rigid-body dynamics, see, e.g., Arribas et al. (2006).

Here we will first summarize the theory of quaternions and then give an overview of the new, elegant way of handling three-dimensional regularization by means of an unconventional conjugation of quaternions, as suggested by Waldvogel (2006a,b). As an application, the well-known theory of Kepler motion will be rederived on the basis of the regularized equations of motion. Furthermore, as a postscriptum to the author's early works (Stiefel and Waldvogel 1965; Waldvogel 1967a,b), the spatial extention of Birkhoff's (1915) regularization of the restricted three-body problem will be elegantly described in terms of quaternions. Finally, we will state the regularized equations of motion of the perturbed spatial Kepler problem.

It seems appropriate that this article appears in the Special Issue in Honor of Claude Frœschlé. It was Claude, with his invitation extended to the author for contributing to the Winter School Les Arcs 2000 on singularities (Benest and Frœschlé 2002), who initiated a process of revisiting regularization theory in celestial mechanics.

\section{Quaternion algebra}

A quote from Wikipedia: Hamilton was looking for ways of extending complex numbers (which can be viewed as points on a two-dimensional plane) to higher spatial dimensions. He could not do so for three dimensions, and in fact it was later shown that it is impossible. Eventually Hamilton tried four dimensions and created quaternions. According to Hamilton, on October 16, 1843 he was out walking along the Royal Canal in Dublin with his wife when the solution in the form of the equations

$$
i^{2}=j^{2}=k^{2}=i j k=-1
$$

suddenly occurred to him; Hamilton then promptly carved these equations into the side of the nearby Broom Bridge. [...] Unfortunately, no trace of the carving remains, though a stone plaque does commemorate the discovery.

Hamilton's basic relations (1) are inconsistent with commutative multiplication rules between the three imaginary units $i, j, k$. However, by postulating commutative multiplication with the real number -1 the better known more explicit multiplication rules may easily be obtained from (1). Right multiplication of the last equality of (1) by $k$ yields $i j=k$; left multiplication of this by $i$ yields $i k=-j$. Furthermore, left multiplication of the last relation of (1) by $k$ and right division by $k$ yields $k i j=-1$. This implies that a cyclic permutation $i \rightarrow j \rightarrow k \rightarrow i$ transforms a valid relation again into a valid relation. Hence 
we obtain the well known non-commutative multiplication rules of the imaginary units:

$$
i j=-j i=k, \quad j k=-k j=i, \quad k i=-i k=j .
$$

Given the real numbers $u_{l} \in \mathbb{R}, l=0,1,2,3$, the object

$$
\mathbf{u}=u_{0}+i u_{1}+j u_{2}+k u_{3}
$$

is called a quaternion $\mathbf{u} \in \mathbb{U}$, where $\mathbb{U}$ denotes the set of all quaternions (in the following bold-face characters denote quaternions). The sum $i u_{1}+j u_{2}+k u_{3}$ is called the quaternion part of $\mathbf{u}$, whereas $u_{0}$ is naturally referred to as its real part. The above multiplication rules and vector space addition define the quaternion algebra. Multiplication is generally non-commutative; however, any quaternion commutes with a real,

$$
c \mathbf{u}=\mathbf{u} c, \quad c \in \mathbb{R}, \quad \mathbf{u} \in \mathbb{U},
$$

and for any three quaternions $\mathbf{u}, \mathbf{v}, \mathbf{w} \in \mathbb{U}$ the associative law holds:

$$
(\mathbf{u v}) \mathbf{w}=\mathbf{u}(\mathbf{v w}) .
$$

The quaternion $\mathbf{u}$ may naturally be associated with the corresponding vector $u=\left(u_{0}, u_{1}, u_{2}, u_{3}\right) \in \mathbb{R}^{4}$. For later reference we introduce notation for 3-vectors in two important particular cases: $\vec{u}=\left(u_{1}, u_{2}, u_{3}\right) \in \mathbb{R}^{3}$ for the vector associated with the pure quaternion $\mathbf{u}=i u_{1}+j u_{2}+k u_{3}$, and $\underline{u}=\left(u_{0}, u_{1}, u_{2}\right)$ for the vector associated with the quaternion having a vanishing $k$-component, $\mathbf{u}=u_{0}+i u_{1}+j u_{2}$.

The conjugate $\overline{\mathbf{u}}$ of the quaternion $\mathbf{u}$ is defined as

$$
\overline{\mathbf{u}}=u_{0}-i u_{1}-j u_{2}-k u_{3} ;
$$

then the modulus $|\mathbf{u}|$ of $\mathbf{u}$ is obtained from

$$
|\mathbf{u}|^{2}=\mathbf{u} \overline{\mathbf{u}}=\overline{\mathbf{u}} \mathbf{u}=\sum_{l=0}^{3} u_{l}^{2} .
$$

As transposition of a product of matrices, conjugation of a quaternion product reverses the order of its factors:

$$
\overline{\mathbf{u v}}=\overline{\mathbf{v}} \overline{\mathbf{u}} .
$$

The two kinds of division by $\mathbf{u} \neq 0$ are carried out by left- or right-multiplication with the inverse $\mathbf{u}^{-1}=\overline{\mathbf{u}} /(\mathbf{u} \overline{\mathbf{u}})$.

A very useful application of quaternions is the possibility of elegantly representing rotations in $\mathbb{R}^{3}$. We only report the result; for a derivation and proof see, e.g., Waldvogel (2006a).

Let $\vec{a}=\left(a_{1}, a_{2}, a_{3}\right) \in \mathbb{R}^{3},|\vec{a}|=1$ be a unit vector defining an oriented rotation axis, and let $\omega$ be a rotation angle. Define the unit quaternion

$$
\mathbf{r}:=\cos \frac{\omega}{2}+\left(i a_{1}+j a_{2}+k a_{3}\right) \sin \frac{\omega}{2} .
$$

Furthermore, let $\vec{x} \in \mathbb{R}^{3}$ be an arbitrary vector, and let $\mathbf{x}=i x_{1}+j x_{2}+k x_{3}$ be the associated pure quaternion. Then the mapping

$$
\mathbf{x} \mapsto \mathbf{y}=\mathbf{r x r}^{-1}
$$

describes the right-handed rotation of $\vec{x}$ about the axis $\vec{a}$ through the angle $\omega$ (since $\mathbf{r}$ is a unit quaternion we have $\mathbf{r}^{-1}=\overline{\mathbf{r}}$ ). 


\section{The KS transformation}

The essential ingredient of regularization in 3-space is the use of a mapping from $\mathbb{R}^{4}$ to $\mathbb{R}^{3}$ that generalizes the conformal squaring used by Levi-Civita (1920) for regularization in the plane. In fact, such a mapping-more precisely, a mapping from the 3-sphere onto the 2-sphere-was discovered already by Hopf (1931) and is referred to in topology as the Hopf mapping.

However, due to the fact that in 3-space only the trivial conformal mappings (translations, rotations and inversions) exist, the possibility of spatial regularization had been missed for a long time. Only in 1964 the use of additional dimensions was considered and finally lead to the now well-known Kustaanheimo-Stiefel or KS regularization. A preliminary version of the KS transformation using spinor notation was proposed by Kustaanheimo (1964); the full theory was developed in a subsequent joint paper (Kustaanheimo and Stiefel 1965); the entire topic is extensively discussed in the comprehensive text by Stiefel and Scheifele (1971).

\subsection{Quaternion representation}

In this subsection we will revisit KS regularization using quaternion notation. As observed by Waldvogel (2006a,b), an elegant and concise representation of the formal computations may be achieved by introducing an unconventional "conjugate", $\mathbf{u}^{\star}$, referred to as the star conjugate of the quaternion $\mathbf{u}=u_{0}+i u_{1}+j u_{2}+k u_{3}$ :

$$
\mathbf{u}^{\star}:=u_{0}+i u_{1}+j u_{2}-k u_{3} .
$$

The star conjugate of $\mathbf{u}$ may be expressed in terms of the conventional conjugate $\overline{\mathbf{u}}$ as

$$
\mathbf{u}^{\star}=k \overline{\mathbf{u}} k^{-1}=-k \overline{\mathbf{u}} k ;
$$

however, it turns out that the definition (11) leads to a particularly elegant treatment of KS regularization. The following elementary properties are easily verified:

$$
\left(\mathbf{u}^{\star}\right)^{\star}=\mathbf{u}, \quad\left|\mathbf{u}^{\star}\right|^{2}=|\mathbf{u}|^{2}, \quad(\mathbf{u v})^{\star}=\mathbf{v}^{\star} \mathbf{u}^{\star} .
$$

Consider now the mapping

$$
\mathbf{u} \in \mathbb{U} \longmapsto \mathbf{x}=\mathbf{u u}^{\star} .
$$

Star conjugation immediately yields $\mathbf{x}^{\star}=\left(\mathbf{u}^{\star}\right)^{\star} \mathbf{u}^{\star}=\mathbf{x}$; hence $\mathbf{x}$ is a quaternion of the form $\mathbf{x}=x_{0}+i x_{1}+j x_{2}$ which may be associated with the vector $\underline{x}=\left(x_{0}, x_{1}, x_{2}\right) \in \mathbb{R}^{3}$. From $\mathbf{u}=u_{0}+i u_{1}+j u_{2}+k u_{3}$ we obtain

$$
\left\{\begin{array}{l}
x_{0}=u_{0}^{2}-u_{1}^{2}-u_{2}^{2}+u_{3}^{2} \\
x_{1}=2\left(u_{0} u_{1}-u_{2} u_{3}\right) \\
x_{2}=2\left(u_{0} u_{2}+u_{1} u_{3}\right),
\end{array}\right.
$$

which is exactly the KS transformation in its classical form or-up to a permutation of the indices - the Hopf mapping. Therefore we have

Theorem 1 The KS transformation $u=\left(u_{0}, u_{1}, u_{2}, u_{3}\right) \in \mathbb{R}^{4} \longmapsto \underline{x}=\left(x_{0}, x_{1}, x_{2}\right) \in \mathbb{R}^{3}$ is given by the quaternion relation

$$
\mathbf{x}=\mathbf{u u}^{\star},
$$

where $\mathbf{u}=u_{0}+i u_{1}+j u_{2}+k u_{3}, \mathbf{x}=x_{0}+i x_{1}+j x_{2}$, and $\mathbf{u}^{\star}$ is defined in (11). 
Corollary 1 The norms of the vectors $\underline{x}$ and $u$ satisfy

$$
r:=\|\underline{x}\|=\|u\|^{2}=\mathbf{u} \overline{\mathbf{u}} .
$$

Proof By appropriately combining the two conjugations and using the rules (13), (5), (7), (8), (12) we obtain

$$
\|\underline{x}\|^{2}=\mathbf{x} \overline{\mathbf{x}}=\mathbf{u}\left(\mathbf{u}^{\star} \overline{\mathbf{u}}^{\star}\right) \overline{\mathbf{u}}=\left|\mathbf{u}^{\star}\right|^{2}|\mathbf{u}|^{2}=|\mathbf{u}|^{4}=\|u\|^{4},
$$

from where the statement follows.

\subsection{Differentiation}

In order to regularize the perturbed 3-dimensional Kepler motion by means of the KS transformation it is necessary to look at the properties of the mapping (13) under differentiation.

The transformation (13) or (14), being a mapping from $\mathbb{R}^{4}$ to $\mathbb{R}^{3}$, leaves one degree of freedom in the parametric space undetermined. In KS theory (Kustaanheimo and Stiefel 1965; Stiefel and Scheifele 1971), this freedom is taken advantage of by trying to inherit as much as possible of the conformality properties of the Levi-Civita mapping, $\mathbf{x}=\mathbf{u}^{2}, \mathbf{x} \in \mathbb{C}, \mathbf{u} \in \mathbb{C}$, but other approaches exist (e.g., Vrbik 1995). By imposing the "bilinear relation"

$$
2\left(u_{3} d u_{0}-u_{2} d u_{1}+u_{1} d u_{2}-u_{0} d u_{3}\right)=0
$$

between the vector $u=\left(u_{0}, u_{1}, u_{2}, u_{3}\right)$ and its differential $d u$ on orbits the tangential mapping of (14) becomes a linear mapping with an orthogonal (but non-normalized) matrix.

This property has a simple consequence on the differentiation of the quaternion representation (13) of the KS transformation. Considering the noncommutativity of the quaternion product, the differential of the mapping (13) becomes

$$
d \mathbf{x}=d \mathbf{u} \cdot \mathbf{u}^{\star}+\mathbf{u} \cdot d \mathbf{u}^{\star},
$$

whereas (16) takes the form of a commutator relation,

$$
\mathbf{u} \cdot d \mathbf{u}^{\star}-d \mathbf{u} \cdot \mathbf{u}^{\star}=0 .
$$

Combining (17) with the relation (18) yields the elegant result

$$
d \mathbf{x}=2 \mathbf{u} \cdot d \mathbf{u}^{\star}
$$

i.e. the bilinear relation (16) of $\mathrm{KS}$ theory is equivalent with the requirement that the tangential mapping of $\mathbf{u} \mapsto \mathbf{u u}^{\star}$ behaves as in a commutative algebra.

\subsection{The inverse mapping}

Since the mapping (14) does not preserve the dimension its inverse in the usual sense does not exist. However, the present quaternion formalism yields an elegant way of finding the corresponding fibration of the original space $\mathbb{R}^{4}$. Being given a quaternion $\mathbf{x}=x_{0}+i x_{1}+j x_{2}$ with a vanishing $k$-component, $\mathbf{x}=\mathbf{x}^{\star}$, we want to find all quaternions $\mathbf{u}$ such that $\mathbf{u} \mathbf{u}^{\star}=\mathbf{x}$. We propose the following solution in two steps:

First step: Find a particular solution $\mathbf{u}:=\mathbf{v}=\mathbf{v}^{\star}=v_{0}+i v_{1}+j v_{2}$ which has a vanishing $k$-component as well. Since $\mathbf{v} \mathbf{v}^{\star}=\mathbf{v}^{2}$ we may obtain $\mathbf{v}$ as one of the quaternion square roots of $\mathbf{x}$, e.g. as

$$
\mathbf{v}=\frac{\mathbf{x}+|\mathbf{x}|}{\sqrt{2\left(x_{0}+|\mathbf{x}|\right)}}
$$


a well-known formula for the square root of the complex number $\mathbf{x}=x_{0}+i x_{1} \in \mathbb{C}$.

Second step: The entire family of solutions (the fibre corresponding to $\mathbf{x}$, geometrically a circle in $\mathbb{R}^{4}$ parametrized by the angle $\varphi$ ), is given by

$$
\mathbf{u}=\mathbf{v} \cdot e^{k \varphi}=\mathbf{v}(\cos \varphi+k \sin \varphi) .
$$

Proof $\mathbf{u u}^{\star}=\mathbf{v} e^{k \varphi} e^{-k \varphi} \mathbf{v}^{\star}=\mathbf{v v}^{\star}=\mathbf{x}$.

\section{Regularization}

In this section we describe the formal procedure for KS-regularizing the equations of motion of the spatial two-body problem by using the four parameters $\left(u_{0}, u_{1}, u_{2}, u_{3}\right)=: u \in \mathbb{R}^{4}$ and quaternion notation, $\mathbf{u}=u_{0}+i u_{1}+j u_{2}+k u_{3}$. The planar case, Levi-Civita (1920), is the particular case $u_{2}=u_{3}=0$, i.e. $\mathbf{u}=u_{0}+i u_{1} \in \mathbb{C}$.

We begin with the differential equations governing the Keplerian motion of a particle about a central body with gravitational parameter $\mu$, written in quaternion notation as

$$
\left.\ddot{\mathbf{x}}+\mu \frac{\mathbf{x}}{r^{3}}=0 \in \mathbb{U}, \quad r=|\mathbf{x}|, \quad \dot{(}\right)=\frac{d}{d t} .
$$

Here $t$ is time, $\underline{x}=\left(x_{0}, x_{1}, x_{2}\right) \in \mathbb{R}^{3}$ is the position of the moving particle, and $\mathbf{x}=$ $x_{0}+i x_{1}+j x_{2} \in \mathbb{U}$ is the corresponding quaternion. In addition, it is necessary to consider the energy integral of (20),

$$
\frac{1}{2}|\dot{\mathbf{x}}|^{2}-\frac{\mu}{r}=-h=\text { const, }
$$

where the right-hand side $-h$ has been chosen such that $h>0$ corresponds to an elliptic orbit.

KS regularization of the spatial Kepler problem may be achieved by the three steps 4.1, 4.2, 4.3 described below. In order to stress the simplicity of this approach we present all the details of the formal computations. Care must be taken to preserve the order of the factors in quaternion products. Exchanging two factors is permitted if one of the factors is real or if the factors are mutually conjugate. An important tool for simplifying expressions is regrouping factors of multiple products according to the associative law (5).

\subsection{First step: slow-motion movie}

This regularization step calls for introducing a new independent variable $\tau$, called fictitious time, according to the Sundman (1907) transformation

$$
d t=r \cdot d \tau, \quad \frac{d}{d \tau}()=()^{\prime} .
$$

Therefore, the ratio $d t / d \tau$ of the two infinitesimal increments is made proportional to the distance $r$; the movie is run in slow-motion whenever $r$ is small. Equations 20, 21 are transformed into

$$
r \mathbf{x}^{\prime \prime}-r^{\prime} \mathbf{x}^{\prime}+\mu \mathbf{x}=0, \quad \frac{1}{2 r^{2}}\left|\mathbf{x}^{\prime}\right|^{2}-\frac{\mu}{r}=-h .
$$


4.2 Second step: conformal squaring with quaternions

The next step of the regularization procedure consists of introducing new coordinates $\mathbf{u} \in \mathbb{U}$ according to the KS or Hopf mapping (13), (14), as a generalization of Levi-Civita's conformal squaring:

$$
\mathbf{x}=\mathbf{u u}^{\star}, \quad r:=|\mathbf{x}|=|\mathbf{u}|^{2}=\mathbf{u} \overline{\mathbf{u}} .
$$

Differentiation by means of (19) yields

$$
\mathbf{x}^{\prime}=2 \mathbf{u u}^{\star^{\prime}}, \quad \mathbf{x}^{\prime \prime}=2 \mathbf{u u}^{\star^{\prime \prime}}+2 \mathbf{u}^{\prime} \mathbf{u}^{\star^{\prime}}, \quad r^{\prime}=\mathbf{u}^{\prime} \overline{\mathbf{u}}+\mathbf{u} \overline{\mathbf{u}}^{\prime} .
$$

Substitution of (24) and (25) into (231) results in the lengthy equation

$$
(\mathbf{u} \overline{\mathbf{u}})\left(2 \mathbf{u} \mathbf{u}^{\star^{\prime \prime}}+2 \mathbf{u}^{\prime} \mathbf{u}^{\star^{\prime}}\right)-\left(\mathbf{u}^{\prime} \overline{\mathbf{u}}+\mathbf{u} \overline{\mathbf{u}}^{\prime}\right) 2 \mathbf{u} \mathbf{u}^{\star^{\prime}}+\mu \mathbf{u} \mathbf{u}^{\star}=0,
$$

which is considerably simplified by observing that the second and third term-after applying the distributive law-compensate:

$$
2(\mathbf{u} \overline{\mathbf{u}}) \mathbf{u}^{\prime} \mathbf{u}^{\star^{\prime}}-2 \mathbf{u}^{\prime}(\overline{\mathbf{u}} \mathbf{u}) \mathbf{u}^{\star^{\prime}}=0 .
$$

Furthermore, by means of (5), (4) and (18) the fourth term of (26) may be simplified as follows:

$$
-2\left(\mathbf{u} \overline{\mathbf{u}}^{\prime}\right)\left(\mathbf{u u}^{\star^{\prime}}\right)=-2 \mathbf{u}\left(\overline{\mathbf{u}}^{\prime} \mathbf{u}^{\prime}\right) \mathbf{u}^{\star}=-2\left|\mathbf{u}^{\prime}\right|^{2} \mathbf{u u}^{\star} .
$$

By using this and left-dividing by $\mathbf{u}$ Eq. 26 now becomes

$$
2 r \mathbf{u}^{\star^{\prime \prime}}+\left(\mu-2\left|\mathbf{u}^{\prime}\right|^{2}\right) \mathbf{u}^{\star}=0 .
$$

4.3 Third step: fixing the energy

From (7), (19), (12) we have

$$
\left|\mathbf{x}^{\prime}\right|^{2}=\mathbf{x}^{\prime} \overline{\mathbf{x}}^{\prime}=4 \mathbf{u}\left(\mathbf{u}^{\star^{\prime}} \overline{\mathbf{u}}^{\star^{\prime}}\right) \overline{\mathbf{u}}=4 r\left|\mathbf{u}^{\prime}\right|^{2}
$$

therefore Eq. $23_{2}$ becomes

$$
\mu-2\left|\mathbf{u}^{\prime}\right|^{2}=r h
$$

Substituting this into the star-conjugate of (27) and dividing by $r$ finally yields

Theorem 2 The KS transformation (13) with the differentiation rule (19) and the time transformation (22) maps the spatial Kepler problem (20) into the quaternion differential equation

$$
2 \mathbf{u}^{\prime \prime}+h \mathbf{u}=0
$$

describing the motion of four uncoupled harmonic oscillators with the common frequency $\omega:=\sqrt{h / 2}$.

\section{The Kepler formulas}

As a first application we present an alternate way of deriving the well-known explicit formulas describing Kepler motion in terms of the eccentric anomaly E. For simplicity 
we restrict ourselves to the planar case $u_{2}=u_{3}=0, \mathbf{u}=u_{0}+i u_{1} \in \mathbb{C}$, in which the KS transformation (13) reduces to Levi-Civita's conformal squaring

$$
\mathbf{x}=x_{0}+i x_{1}=\mathbf{u}^{2} \text {. }
$$

The differential equation (20) of Kepler motion,

$$
\ddot{\mathbf{x}}+\mu \frac{\mathbf{x}}{r^{3}}=0 \in \mathbb{C}, \quad r=|\mathbf{x}|=|\mathbf{u}|^{2}
$$

is transformed into (30),

$$
2 \mathbf{u}^{\prime \prime}+h \mathbf{u}=0 \in \mathbb{C} \quad \text { with } \quad d t=r d \tau, \quad h=-\frac{1}{2}|\dot{\mathbf{x}}|^{2}+\frac{\mu}{r}=\text { const }>0 .
$$

In this section bold-face characters denote complex numbers.

We begin with the general solution of (30) in two dimensions,

$$
\mathbf{u}=A \cos (\omega \tau)+i B \sin (\omega \tau) \in \mathbb{C}, \quad \omega=\sqrt{h / 2},
$$

thus parametrizing the origin-centered elliptic orbit of a planar harmonic oscillator by means of $\tau$. For simplicity we assume $A, B \in \mathbb{R}$; this corresponds to using a coordinate system aligned with the principal axes of the orbit. Then $\tau=0$ corresponds to an apex of the ellipse.

\subsection{The eccentric anomaly}

The square of (32),

$$
\mathbf{x}=\mathbf{u}^{2}=\frac{A^{2}-B^{2}}{2}+\frac{A^{2}+B^{2}}{2} \cos (2 \omega \tau)+i A B \sin (2 \omega \tau),
$$

describes the elliptic Keplerian orbit of Fig. 1. By comparing the figure with Eq. 33 the geometric meaning of the angle

$$
E:=2 \omega \tau=\sqrt{2 h} \tau,
$$

may immediately be identified as the angle marked in Fig. 1, having its vertex at the center of the ellipse. $E$ is referred to as the eccentric anomaly of the Kepler motion under consideration; it is known to be the ideal parameter for describing Kepler motion. In the present approach it comes into play in a completely natural way.

\subsection{The orbit}

From (33) and Fig. 1 we immediately identify the geometric parameters $a, b$ (major and minor semi-axes), and $c$ (distance of the center from the origin) as

$$
a=\frac{A^{2}+B^{2}}{2}, \quad b=A B, \quad c=\frac{A^{2}-B^{2}}{2} .
$$

Because of $c^{2}+b^{2}=a^{2}$ the origin is a focus of the ellipse; therefore the eccentricity is

$$
e:=\frac{c}{a}=\frac{A^{2}-B^{2}}{A^{2}+B^{2}} .
$$

In terms of $a, e$ the parameters $A, B$ may now be written as

$$
A=\sqrt{a(1+e)}, \quad B=\sqrt{a(1-e)} .
$$


Fig. 1 The planar elliptic Kepler motion with eccentricity $e=0.9$. $a, b$ semi- axes, $c$ focal distance, $p$ semi-latus rectum, $E$ eccentric anomaly, $\mu$ gravitational parameter, $r$ distance, $\phi$ polar angle

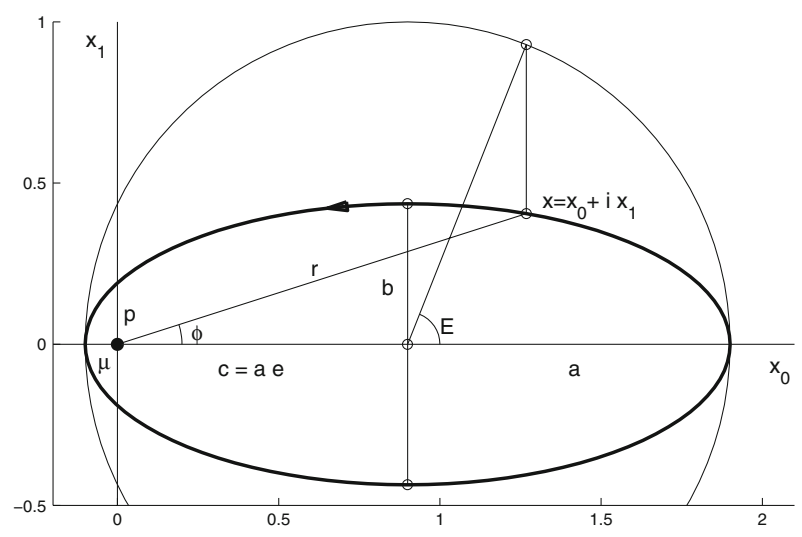

Therefore, the parametrization of the orbit (33) in terms of $E$, in view of $\mathbf{x}=x_{0}+i x_{1}$, becomes

$$
x_{0}=a(e+\cos (E)), \quad x_{1}=a \sqrt{1-e^{2}} \sin (E) .
$$

Furthermore, by using (32), (35) and (36) the distance $r$ is found to be

$$
r=|\mathbf{x}|=|\mathbf{u}|^{2}=\frac{A^{2}+B^{2}}{2}+\frac{A^{2}-B^{2}}{2} \cos (E)=a(1+e \cos (E)) .
$$

\subsection{Energy}

According to Eq. 21 the negative energy $h$ is a constant of motion. In this section we will establish a relationship between $h$ and the major semi-axis $a$. According to (29) and (39) we have

$$
2\left|\mathbf{u}^{\prime}\right|^{2}=\mu-r h \quad \text { with } \quad r=a(1+e \cos (E)) .
$$

On the other hand, the derivative of the regularized orbit (32) implies the relation $\left|\mathbf{u}^{\prime}\right|^{2}=$ $\omega^{2} a(1-e \cos (E))$ or

$$
2\left|\mathbf{u}^{\prime}\right|^{2}=a h(1-e \cos (E)) .
$$

This is compatible with (40) for every $E$ if and only if

$$
2 a h=\mu \text { or } h=\frac{\mu}{2 a} .
$$

\subsection{Time}

The motion as a function of time easily follows by rewriting Sundman's transformation (22) in terms of $E$ by means of (34) and (39):

$$
d t=\frac{a}{\sqrt{2 h}}(1+e \cos (E)) d E .
$$

By using (41) this becomes

$$
d t=\frac{1}{n}(1+e \cos (E)) d E \quad \text { with } \quad n:=\sqrt{\frac{\mu}{a^{3}}} .
$$


The quantity $n=2 \pi / T$ ( $T$ the period of revolution) is the mean angular velocity of the particle, or mean motion, as it is called in astronomy. Finally, integration of $\left(42_{1}\right)$ (normalized for $t=0$ at the apocenter) yields Kepler's equation

$$
t=\frac{1}{n}(E+e \sin (E))
$$

whereas $\left(42_{2}\right)$ is Kepler's third law,

$$
n^{2} a^{3}=\mu
$$

\subsection{Polar coordinates}

Keplerian orbits have a surprisingly simple representation in polar coordinates $r, \phi$ satisfying $\mathbf{x}=r e^{i \phi}$. Rewriting (32) in terms of $E, a, e$ by means of (34), (37) yields

$$
\mathbf{u}=\sqrt{\mathbf{x}}=\sqrt{a(1+e)} \cos \left(\frac{E}{2}\right)+i \sqrt{a(1-e)} \sin \left(\frac{E}{2}\right)=\sqrt{r} e^{i \phi / 2} .
$$

This immediately implies the famous relation

$$
\tan \left(\frac{\phi}{2}\right)=\sqrt{\frac{1-e}{1+e}} \tan \left(\frac{E}{2}\right)
$$

Solving (45) for $\tan (E / 2)$ and passing over to $\cos (E)$ yields

$$
\cos (E)=\frac{\cos (\phi)-e}{1-e \cos (\phi)}
$$

Substituting this into the last expression for $r$ in (39) yields

$$
r=\frac{p}{1-e \cos (\phi)} \quad \text { with } \quad p=a\left(1-e^{2}\right) .
$$

$p$ is called the semi-latus rectum; it is the value of $r$ for $\phi=\pi / 2$.

\subsection{Angular momentum}

The invariance of the angular momentum vector $\underline{D}$ may be derived directly from the equations of motion (20) by considering the vector product $\underline{D}=\underline{x} \times \underline{\dot{x}}$. Following the philosophy of this section, we will derive the property from the orbit by explicit computations.

Again restricting ourselves to the planar case and using the complex position $\mathbf{x}=x_{0}+i x_{1}$, the scalar angular momentum of a particle of unit mass becomes $D=\operatorname{Im}(\overline{\mathbf{x}} \dot{\mathbf{x}})$. By using the orbit (38) as well as $r$ from (39) and $p$ from (46) we obtain

$$
\operatorname{Im}\left(\overline{\mathbf{x}} \frac{d \mathbf{x}}{d E}\right)=\sqrt{a p} \cdot r
$$

Transforming this to time derivatives by means of (42) yields

$$
D=\operatorname{Im}\left(\overline{\mathbf{x}} \frac{d \mathbf{x}}{d t}\right)=\sqrt{\mu p}=\text { const. }
$$




\section{The Birkhoff transformation}

The conformal mapping proposed by Birkhoff (1915) regularizes all singularities of the planar restricted three-body problem with a single transformation. The same transformation - under the name Joukowsky mapping — is being used in aerodynamics in order to map the cross section of airfoils to near-circular domains. A three-dimensional generalization on the basis of the KS transformation was discovered by Stiefel and Waldvogel (1965). Later these ideas were used by Waldvogel (1967a,b).

As a second application of our quaternion formalism for regularization we will summarize the spatial generalization of the Joukowsky-Birkhoff transformation, following Waldvogel (2006b). The theory developed in Sects. 2-4 allows for an elegant representation of the spatial Birkhoff mapping. A concise proof of the resulting transformation equation will be added.

We begin by revisiting the classical (planar) Birkhoff transformation and represent it as the composition of three elementary conformal mappings; this will then readily generalize to the spatial situation by means of quaternions.

Consider a rotating physical plane parametrized by the complex variable $\mathbf{y} \in \mathbb{C}$; for convenience we assume the fixed primaries of the restricted three-body problem to be located at the points A, C given by the complex posititons $\mathbf{y}=-1$ and $\mathbf{y}=1$, respectively (see Fig. 2). The complex variable of the parametric plane will be denoted by $\mathbf{v}$ and will be normalized in such a way that the primaries correspond to $\mathbf{v}=-1$ or $\mathbf{v}=1$, respectively.

The key observation is that Levi-Civita's conformal mapping (31), $\mathbf{u} \mapsto \mathbf{x}=\mathbf{u}^{2}$, not only regularizes collisions at $\mathbf{x}=0$ but also analogous singularities at $\mathbf{x}=\infty$. This is seen by closing the complex planes to become Riemann spheres (by adding the point at infinity) and using inversions $\mathbf{x}=1 / \tilde{\mathbf{x}}, \mathbf{u}=1 / \tilde{\mathbf{u}}$.

Taking advantage of this fact, we first map the $\mathbf{v}$-sphere to an auxiliary $\mathbf{u}$-sphere by the Möbius transformation

$$
\mathbf{v} \longmapsto \mathbf{u}=\frac{\mathbf{v}+1}{\mathbf{v}-1}=1+\frac{2}{\mathbf{v}-1}
$$

which takes the primaries A, C to the points $\mathbf{u}=0, \mathbf{u}=\infty$, respectively. The Levi-Civita mapping (31) will leave these points invariant while regularizing collisions at A or C. Finally, the Möbius transformation

$$
\mathbf{x} \longmapsto \mathbf{y}=\frac{\mathbf{x}+1}{\mathbf{x}-1}=1+\frac{2}{\mathbf{x}-1}
$$
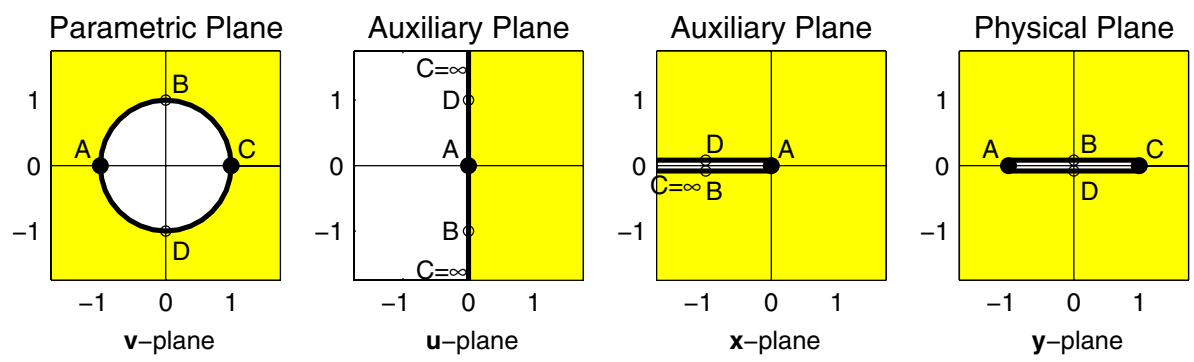

$$
\mathbf{v} \mapsto \mathbf{u}=\frac{\mathbf{v}+1}{\mathrm{v}-1} \quad \mathbf{u} \mapsto \mathbf{x}=\mathbf{u}^{2} \quad \mathrm{x} \mapsto \mathbf{y}=\frac{\mathrm{x}+1}{\mathrm{x}-1}
$$

Fig. 2 The sequence of conformal mappings generating the planar Birkhoff transformation 
maps A, C to $\mathbf{y}=-1$ and $\mathbf{y}=1$, respectively. The composition of the mappings (48), (31), (49) yields

$$
\mathbf{y}=\frac{\left(\frac{\mathbf{v}+1}{\mathbf{v}-1}\right)^{2}+1}{\left(\frac{\mathbf{v}+1}{\mathbf{v}-1}\right)^{2}-1} \text { or } \mathbf{y}=\frac{1}{2}\left(\mathbf{v}+\frac{1}{\mathbf{v}}\right),
$$

the well known mapping used by Joukowsky (1847-1921) and by G. D. Birkhoff (18841944).

In the spatial case we choose $\mathbf{v}, \mathbf{u}, \mathbf{x}, \mathbf{y} \in \mathbb{U}$ to be quaternions, $\mathbf{x}=\mathbf{x}^{\star}, \mathbf{y}=\mathbf{y}^{\star}$ being quaternions with vanishing $k$-components associated with 3 -vectors $\underline{x}, y$. Then the mappings (48), (49), now being shifted inversions in four or three dimensions, are both conformal mappings, in fact the only nontrivial conformal mappings existing in those dimensions (except for rotated versions). Composing these with the KS or Hopf mapping (13), $\mathbf{u} \mapsto \mathbf{x}=\mathbf{u u}^{\star}$, yields

Theorem $\mathbf{3}$ Let $\mathbf{v} \in \mathbb{U}$ be the quaternion coordinate in a four-dimensional parametric space $\mathbb{R}^{4}$, such that the points $\mathbf{v}= \pm 1$ correspond to the positions of the primaries of a spatial restricted three-body problem. Then

$$
\mathbf{y}=1+\left(\mathbf{v}^{\star}-1\right)\left(\mathbf{v}+\mathbf{v}^{\star}\right)^{-1}(\mathbf{v}-1)
$$

generalizes the Joukowsky-Birkhoff mapping from $\mathbb{R}^{4}$ to $\mathbb{R}^{3}$ with quaternion coordinates $\mathbf{y}=\mathbf{y}^{\star}=y_{0}+i y_{1}+j y_{2} \in \mathbb{U}$, where the primaries are normalized to be located at $\mathbf{y}= \pm 1$.

Remark The right-hand side of (51) is easily split up into components by means of the inversion formula $\mathbf{u}^{-1}=\overline{\mathbf{u}} /|\mathbf{u}|^{2}$ of Sect. 2; they agree with the results of Stiefel and Waldvogel (1965) up to the sign of $v_{3}$. Both transformations regularize; the discrepancy is due to a different definition of the orientation in the inversions.

Proof Composition of (49) with (13) and (48) (in the appropriate quaternion versions) yields

$$
\mathbf{y}=1+2\left(\mathbf{u u}^{\star}-1\right)^{-1} \quad \text { with } \quad \mathbf{u}=1+2(\mathbf{v}-1)^{-1} .
$$

Rewriting $\mathbf{u u}^{\star}-1$ as

$$
\mathbf{u u}^{\star}-1=(\mathbf{u}-1)\left(\mathbf{u}^{\star}-1\right)+\mathbf{u}-1+\mathbf{u}^{\star}-1
$$

and substituting $\mathbf{u}$ from $\left(52_{2}\right)$ yields

$$
\mathbf{u u}^{\star}-1=4(\mathbf{v}-1)^{-1}\left(\mathbf{v}^{\star}-1\right)^{-1}+2(\mathbf{v}-1)^{-1}+2\left(\mathbf{v}^{\star}-1\right)^{-1} .
$$

By inserting appropriate unit factors, Eq. 52 1 becomes

$$
\mathbf{y}=1+2\left(\mathbf{v}^{\star}-1\right) \underbrace{\left(\mathbf{v}^{\star}-1\right)^{-1}\left(\mathbf{u u}^{\star}-1\right)^{-1}(\mathbf{v}-1)^{-1}}_{\mathbf{D}^{-1}}(\mathbf{v}-1) .
$$

Introducing the "denominator" $\mathbf{D}$ by defining $\mathbf{D}^{-1}$ as indicated in (54) we obtain

$$
\mathbf{D}=(\mathbf{v}-1)\left(\mathbf{u u}^{\star}-1\right)\left(\mathbf{v}^{\star}-1\right),
$$

which, by using (53), simplifies to

$$
\mathbf{D}=2\left(\mathbf{v}+\mathbf{v}^{\star}\right) \text {. }
$$

Now the statement (51) of Theorem 3 follows directly from (54). 


\section{The perturbed Kepler problem}

Our third application of quaternion regularization is the perturbed spatial Kepler problem,

$$
\ddot{\mathbf{x}}+\mu \frac{\mathbf{x}}{r^{3}}=\varepsilon \mathbf{f}(\mathbf{x}, t), \quad r=|\mathbf{x}|,
$$

written in quaternion notation. $\mathbf{f}(\mathbf{x}, t)$ is the perturbing function, $\mathbf{x} \in \mathbb{U}$ and $\mathbf{f} \in \mathbb{U}$ are quaternions with vanishing $k$-components, and $\varepsilon$ is a small parameter. Note that in the perturbed case an energy equation formally identical with (21) still holds. However, $h=h(t)$ and $a=a(t)$ are now slowly varying functions of time, $a(t)$ being the osculating major semi-axis; $h(t)$ satisfies the differential equaion

$$
\dot{h}=-\langle\underline{\dot{x}}, \varepsilon \underline{f}\rangle \text { or } h^{\prime}=-\left\langle\underline{x^{\prime}}, \varepsilon \underline{f}\right\rangle,
$$

where $\langle\cdot, \cdot\rangle$ denotes the dot product of 3 -vectors.

In the following, we report the results of the regularization procedure outlined in Sect. 4; the details are left to the reader. Step 1 yields

$$
r \mathbf{x}^{\prime \prime}-r^{\prime} \mathbf{x}^{\prime}+\mu \mathbf{x}=r^{3} \varepsilon \mathbf{f}(\mathbf{x}, t)
$$

instead of Eq. 231. By using (28) the energy equation (29) again becomes

$$
\mu-2\left|\mathbf{u}^{\prime}\right|^{2}=r h .
$$

The right-hand side of Eq. 26 becomes

$$
\mathbf{u} \overline{\mathbf{u}} r^{2} \varepsilon \mathbf{f}(\mathbf{x}, t)
$$

instead of 0. Simplification as in Sect. 4 as well as left-multiplication by $r^{-1} \mathbf{u}^{-1}$ and star conjugation finally yields the perturbing equation for the quaternion coordinate $\mathbf{u}$ :

Theorem 4 KS regularization, as formulated in terms of quaternions in Sect. 4, transforms the perturbed Kepler problem (56) into the perturbed harmonic oscillator

$$
2 \mathbf{u}^{\prime \prime}+h \mathbf{u}=r \varepsilon \mathbf{f}(\mathbf{x}, t) \overline{\mathbf{u}}^{\star}, \quad r=|\mathbf{u}|^{2},
$$

where $h=r^{-1}\left(\mu-2\left|\mathbf{u}^{\prime}\right|^{2}\right)$ is the negative of the (slowly varying) energy.

In the following summary we collect the complete set of differential equations defining the regularized system equivalent to the perturbed spatial Kepler problem (56). The harmonic oscillator of Theorem 4 appears in the first line. For stating an initial-value problem a starting value of $\mathbf{u}$ needs to be chosen according to Sect. 3.3. The corresponding initial velocity is obtained by solving (19) for $d \mathbf{u}$ :

$$
\frac{d \mathbf{u}}{d \tau}=\frac{1}{2 r} \frac{d \mathbf{x}}{d t} \overline{\mathbf{u}}^{\star}
$$

Summary Regularized system corresponding to the perturbed spatial Kepler problem (56):

$$
\begin{cases}2 \mathbf{u}^{\prime \prime}+h \mathbf{u}=\operatorname{r\varepsilon } \mathbf{f}(\mathbf{x}, t) \overline{\mathbf{u}}^{\star}, & r=|\mathbf{u}|^{2}, \quad()^{\prime}=\frac{d}{d \tau} \\ t^{\prime}=r, & \mathbf{x}=\mathbf{u u}^{\star} \\ h^{\prime}=-\varepsilon\left\langle\underline{x}^{\prime}, \underline{f}(\mathbf{x}, t)\right\rangle & \text { or } h=r^{-1}\left(\mu-2\left|\mathbf{u}^{\prime}\right|^{2}\right) .\end{cases}
$$


Remark Introducing the osculating eccentric anomaly $E$ by the differential relation $d E=$ $\sqrt{2 h} d \tau$ transforms the first equation of (60) into

$$
4 \mathbf{u}^{\prime \prime}+\mathbf{u}=\frac{\varepsilon}{h}\left(r \mathbf{f}(\mathbf{x}, t) \overline{\mathbf{u}}^{\star}+2\left\langle\underline{x}^{\prime}, \underline{f}(\mathbf{x}, t)\right\rangle \mathbf{u}^{\prime}\right),
$$

a perturbed harmonic oscillator with constant frequency $\omega=\frac{1}{2}$. Here ()$^{\prime}=d / d E$. This equation is particularly well suited for introducing orbital elements with simple pertubation equations by means of the method of varying the constant, see, e.g., Waldvogel (2006a).

\section{References}

Arribas, M., Elipe, A., Palacios, M.: Quaternions and the rotation of a rigid body. Celest. Mech. Dyn. Astron. 96, 239-251 (2006)

Birkhoff, G.D.: The restricted problem of three bodies. Rendiconti del Circolo Matematico di Palermo 39, 1 (1915); Reprinted in Collected Mathematical Papers, vol. 1. Dover Publications, New York (1968)

Benest, D., Frœschlé, C. (eds.): Singularities in Gravitational Systems, 215 pp. Lecture Notes in Physics. Springer-Verlag, Berlin (2002)

Chelnokov, Y.N.: On regularization of the equations of the three-dimensional two-body problem. Izv. Akad. Nauk SSSR, Ser. Mekh. Tverd. Tela [Mechanics of Solids], 12-21 (1981); 151-158 (1984)

Chelnokov, Y.N.: Application of quaternions in the theory of orbital motion of a satellite. Kosm. Issled. [Cosmic Res.] 30, 759-770 (1992); 31, 3-15 (1993)

Chelnokov, Y.N.: Application of quaternions in the mechanics of space flight. Gyroscopy Navigation 4(27), 47-66 (1999)

Hamilton, W.R.: On quaternions, or a new system of imaginaries in algebra. Philos. Mag. 25, 489-495 (1844)

Hopf, H.: Über die Abbildung der dreidimensionalen Sphäre auf die Kugelfläche. Math. Ann. 104 (1931). Reprinted in Selecta Heinz Hopf, pp. 38-63. Springer-Verlag, Berlin (1964)

Kustaanheimo, P.: Spinor regularisation of the Kepler motion. Ann. Univers. Turkuensis, Ser. A, 73, 1-7 (1964); Publ. Astr. Obs. Helsinki 102

Kustaanheimo, P., Stiefel, E.L.: Perturbation theory of Kepler motion based on spinor regularization. J. Reine Angew. Math. 218, 204-219 (1965)

Levi-Civita, T.: Sur la régularisation du problème des trois corps. Acta Math. 42, 99-144 (1920)

Stiefel, E.L., Scheifele, G.: Linear and Regular Celestial Mechanics, 301 pp. Springer-Verlag, Berlin (1971)

Stiefel, E.L., Waldvogel, J.: Généralisation de la régularisation de Birkhoff pour le mouvement du mobile dans l'espace à trois dimensions. C.R. Acad. Sci. Paris 260, 805 (1965)

Sundman, K.F.: Recherches sur le problème des trois corps. Acta Societatis Scientificae Fennicae 34, 6 (1907) Vivarelli, M.D.: The KS transformation in hypercomplex form. Celest. Mech. Dyn. Astron. 29, 45-50 (1983)

Vrbik, J.: Celestial mechanics via quaternions. Can. J. Phys. 72, 141-146 (1994)

Vrbik, J.: Perturbed Kepler problem in quaternionic form. J. Phys. A 28, 193-198 (1995)

Waldvogel, J.: Die Verallgemeinerung der Birkhoff-Regularisierung für das räumliche Dreikörperproblem. Bulletin Astronomique, Série 3, Tome II, Fasc. 2, 295-341 (1967a)

Waldvogel, J.: The restricted elliptic three-body problem. In: Stiefel E. et al. (eds.) Methods of Regularization for Computing Orbits in Celestial Mechanics. NASA Contractor Report NASA CR, vol. 769, pp. 88-115 (1967b)

Waldvogel, J.: Order and chaos in satellite encounters. In Steves, B.A. et al. (eds.) Chaotic Worlds: From Order to Disorder in Gravitational N-Body Dynamical Systems, pp. 233-254. Springer, Dordrecht (2006a)

Waldvogel, J.: Quaternions and the perturbed Kepler problem. Celest. Mech. Dyn. Astron. 95, 201-212 (2006b) 